\title{
Sources and propagation of errors in quantitative phase imaging techniques using optical interferometry
}

Manuel Bedrossian, Jay Nadeau, Eugene Serabyn, Chris Lindensmith

Manuel Bedrossian, Jay Nadeau, Eugene Serabyn, Chris Lindensmith, "Sources and propagation of errors in quantitative phase imaging techniques using optical interferometry," Proc. SPIE 10074, Quantitative Phase Imaging III, 100740E (21 February 2017); doi: 10.1117/12.2250069

SPIE. Event: SPIE BiOS, 2017, San Francisco, California, United States 


\title{
Sources and Propagation of Errors in Quantitative Phase Imaging Techniques using Optical Interferometry
}

\author{
${ }^{1}$ Department of Medical Engineering. California Institute of Technology. 1200 E. California Blvd. Pasadena, CA 91125. USA \\ ${ }^{2}$ Jet Propulsion Laboratory. Californian Institute of Technology. 4800 Oak Grove Dr. Pasadena, CA 91107. USA
}

\begin{abstract}
Quantitative phase imaging (QPI) has many applications in a broad range of disciplines from astronomy to microbiology. QPI is often performed by optical interferometry, where two coherent beams of light are used to produce interference patterns at a detector plane. Many algorithms exist to calculate the phase of the incident light from these recorded interference patterns as well as enhance their quality by various de-noising methods. Many of these de-noising algorithms, however, corrupt the quantitative aspect of the measurement, resulting in phase contrast images. Among these phase calculation techniques and de-noising algorithms, none approach the optimization of phase measurements by theoretically addressing the various sources of error in its measurement, as well as how these errors propagate to the phase calculations. In this work, we investigate the various sources of error in the measurements required for QPI, as well as theoretically derive the influence of each source of error on the overall phase calculation for three common phase calculation techniques: the four bucket/step method, three bucket/step method, and the Carré method. The noise characteristics of each of these techniques are discussed and compared using error parameters of a readily available CCD sensor array. Additionally, experimental analysis is conducted on interferograms to investigate the influence of speckle noise on the phase measurements of the three algorithms discussed.
\end{abstract}

\section{INTRODUCTION}

Quantitative phase imaging is becoming an increasingly common tool used in many academic and research disciplines, including astronomy and biological microscopy. The use of interferometry for the measurement of angular sizes and separation of astronomical objects was suggested by Armand Hippolyte Louis Fizeau in 1868, but due to challenges related to its practical implementation, it did not become a widely-used method until the 1950s to 1970s[1]. Phase contrast microscopy was developed in the 1930s, and remains an important technique in light microscopy[2, 3]. However, phase contrast does not provide quantitative information about the phase shift produced by the object. Methods of quantifying this shift are referred to as quantitative phase imaging (QPI) or quantitative phase microscopy, and are implemented using a variety of interferometric techniques[4-6].

Interferometry exploits the wave nature of light in order to create interference between two monochromatic beams. An object in the optical path of one of these beams will distort the wavefront, which results in a change to the original interference pattern. This change in the interference pattern, or fringe, is used to calculate the phase shift introduced in the light by the object[7]. Many algorithms have been developed to extract phase information from these interference patterns such as the Four Bucket/Step Method, Three Bucket/Step Method, and the Carré Method. These techniques vary in the spatial/temporal sampling frequency of the interference patterns, which dictates the expression used to calculate phase[7].

Due to the optical setup required in most interferometric devices, the measurement of phase introduced by an object is highly susceptible to errors. These errors are in the form of vibrations that cause misalignments of the two beams, laser speckle, temporal phase noise, errors due to uncorrelated noise between the two arms of the interferometer as well as error introduced by the detector used to record the interference pattern.

Both pre and post-processing algorithms exist to minimize these errors. The interferometric setup may be optimized by reducing the recording distance and using a detector with smaller pixel sizes[8]. Other methods to reduce noise include phase error compensation, spatial light modulation (SLM), and multiple frequency overlapping[9] [10-13], which improve the contrast of phase measurements. Noise reduction is also accomplished by filtering certain frequencies, both in the spatial and Fourier planes, with Butterworth filters and masks, respectively[14, 15] [6]. In addition, efficient

Quantitative Phase Imaging III, edited by Gabriel Popescu, YongKeun Park, Proc. of SPIE Vol.

10074, 100740E · C 2017 SPIE · CCC code: 1605-7422/17/\$18 · doi: 10.1117/12.2250069 
encoding methods and correlation based de-noising algorithms have been developed to significantly reduce speckle noise[16, 17]. During biological studies, the power of the light source must sometimes be monitored as to not harm the organisms being observed. This has prompted investigation into the reduction of errors introduced by shot noise that becomes significant at low illumination[18, 19].

Although the aforementioned de-noising algorithms are effective, a majority of them corrupt the quantitative nature of interferometric phase measurements and result in enhanced phase contrast imaging. These experimental approaches to address and minimize phase errors during interferometric measurements do not address the separate sources of error within a phase measurement, nor do they address the manner in which these sources of error propagate to the final phase calculation

In this work, we first review the theoretical background of interferometry, and three common phase calculation algorithms, as well as perform an error analysis on these phase calculation techniques. The separate sources of error considered are photon noise, detector noise, and quantization noise. The propagation of these sources of error are investigated showing their influence in the resulting phase calculation for each phase technique. From the error analyses, simulations were performed using performance parameters of a readily available detector array (CCD) in order to compare and contrast the performance of each phase calculation technique. Experimental data of interferograms are also investigated to quantify the effects of speckle noise introduced by the coherent nature of the illumination source needed to produce interference patterns at the detector.

\section{THEORY}

Quantitative phase imaging is performed by the use of optical interferometry. This technique encodes both the amplitude and phase characteristics of an object. It does this with the superposition of a reference and object light beam, which are monochromatic and coherent. The reference and object beam are recombined at the detector plane which then creates interference patterns, or an interferogram. The beams of an interferometer can be represented as plane waves of identical wavelengths, such that their displacement functions are:

$$
\begin{aligned}
& \psi_{1}(x, y, t)=A_{1}(x, y) e^{i\left(\phi_{1}(x, y)-\omega t\right)} \\
& \psi_{2}(x, y, t)=A_{2}(x, y) e^{i\left(\phi_{2}(x, y)-\omega t\right)}
\end{aligned}
$$

Where $A_{1}$ and $A_{2}$ are the amplitudes of the electric field as a function of $x$ and $y, \phi_{1}$ and $\phi_{2}$ are relative phases of waves 1 and 2 respectively, and $\omega$ is the angular frequency of the wave.

The combination of these two waves at the detector plane causes the resultant wave to be expressed using the superposition principle such that:

$$
\boldsymbol{\Psi}(x, y)=\psi_{1}+\psi_{2}=A_{1}(x, y) e^{i\left(\phi_{1}(x, y)-\omega t\right)}+A_{2}(x, y) e^{i\left(\phi_{2}(x, y)-\omega t\right)}
$$

Modern optical detector arrays' mode of operation is by the integration of the electric field incident on it over a period of time. The measured electric field by the detector becomes:

$$
I(x, y)=\int_{t_{1}}^{t_{2}} \boldsymbol{\Psi} \boldsymbol{\Psi}^{*} d t=I_{1}+I_{2}+2 \sqrt{I_{1} I_{2}} \cos (\Delta \phi)
$$

where $\boldsymbol{\Psi}^{*}$ is the complex conjugate of $\boldsymbol{\Psi}, \Delta \phi$ is the phase difference between the two waves, and $I_{1}$ and $I_{2}$ are the intensities of beams 1 and 2 , respectively.

With the ability to measure the electric field caused by the superposition of two waves, it is possible to calculate the phase of light at each point of the detector. There are many algorithms that can accomplish this; the three techniques that will be discussed in this work are the Four Bucket/Step Method, the Three Bucket/Step Method, and the Carré Method. 


\section{Four Bucket/Step Method}

A common algorithm for the extraction of quantitative phase information from such interferograms, or fringes, is called the 'Four Step' or 'Four Bucket' Method, where a single fringe is sampled four times along its length. These four points can be sampled at discrete points in space or time, corresponding to a Four Bucket or Four Step method, respectively.

In the Four Bucket method, a time invariant interference pattern is sampled across four adjacent pixels that have a phase offset of $\pi / 2$ from each other. Similarly, in a Four Step method, a single pixel experiences a phase step as a function of time such that the light incident on it from exposure $i$ to $i+1$ carries of phase offset of $\pi / 2$.

According to Equation 3, the intensities of the electric field at each of the four sample points are:

$$
\begin{gathered}
I_{a}=I_{1}+I_{2}+2 \sqrt{I_{1} I_{2}} \cos (\Delta \phi) \\
I_{b}=I_{1}+I_{2}+2 \sqrt{I_{1} I_{2}} \cos \left(\Delta \phi+\frac{\pi}{2}\right)=I_{1}+I_{2}-2 \sqrt{I_{1} I_{2}} \sin (\Delta \phi) \\
I_{c}=I_{1}+I_{2}+2 \sqrt{I_{1} I_{2}} \cos (\Delta \phi+\pi)=I_{1}+I_{2}-2 \sqrt{I_{1} I_{2}} \cos (\Delta \phi) \\
I_{d}=I_{1}+I_{2}+2 \sqrt{I_{1} I_{2}} \cos \left(\Delta \phi+\frac{3 \pi}{2}\right)=I_{1}+I_{2}+2 \sqrt{I_{1} I_{2}} \sin (\Delta \phi)
\end{gathered}
$$

With these equations describing the electric fields of the incident light, phase $(\Delta \phi)$ becomes:

$$
\Delta \phi=\arctan \left(\frac{\sin (\Delta \phi)}{\cos (\Delta \phi)}\right)=\arctan \left(\frac{I_{d}-I_{b}}{I_{a}-I_{c}}\right)
$$

\section{Three Bucket/Step Method}

The Three Bucket/Step Method requires three data points (exposures) in order to calculate the phase of the wavefront. This is accomplished by sampling the fringe pattern at three predefined phase offsets. A common phase offset used is $\pi / 2$ from each subsequent exposure. Other phase offsets may be used, such as $2 \pi / 3$ [7]. The intensities at each exposure can then be expressed as:

$$
\begin{gathered}
I_{a}=I_{1}+I_{2}+2 \sqrt{I_{1} I_{2}} \cos (\Delta \phi) \\
I_{b}=I_{1}+I_{2}+2 \sqrt{I_{1} I_{2}} \cos \left(\Delta \phi+\frac{\pi}{2}\right)=I_{1}+I_{2}-2 \sqrt{I_{1} I_{2}} \sin (\Delta \phi) \\
I_{c}=I_{1}+I_{2}+2 \sqrt{I_{1} I_{2}} \cos (\Delta \phi+\pi)=I_{1}+I_{2}-2 \sqrt{I_{1} I_{2}} \cos (\Delta \phi)
\end{gathered}
$$

With these equations describing the electric field of an interference pattern, the phase difference can be calculated as:

$$
\Delta \phi=\arctan \left(\frac{\sin \Delta \phi}{\cos \Delta \phi}\right)=\arctan \left(\frac{I_{c}-I_{b}}{I_{a}-I_{b}}\right)
$$

\section{Carré Method}

Unlike the four or three bucket/step methods that use a fixed and predefined phase offset between exposures, the Carré Method allows for phase calculations where the phase offset between exposures can vary linearly. This requires four exposures, each with a phase offset of $\alpha$ between each exposure, where $\alpha \in(0, \pi]$. The four resulting intensities can be expressed as:

$$
\begin{gathered}
I_{a}=I_{1}+I_{2}+2 \sqrt{I_{1} I_{2}} \cos (\Delta \phi-3 \alpha / 2) \\
I_{a}=I_{1}+I_{2}+2 \sqrt{I_{1} I_{2}} \cos (\Delta \phi-\alpha / 2) \\
I_{a}=I_{1}+I_{2}+2 \sqrt{I_{1} I_{2}} \cos (\Delta \phi+\alpha / 2) \\
I_{a}=I_{1}+I_{2}+2 \sqrt{I_{1} I_{2}} \cos (\Delta \phi+3 \alpha / 2)
\end{gathered}
$$


The unknown phase shift between exposures $(\alpha)$ is calculated using the following expression:

$$
\alpha=2 \arctan \left(\sqrt{\frac{3\left(I_{b}-I_{c}\right)-\left(I_{a}-I_{c}\right)}{\left(I_{b}+I_{c}\right)-\left(I_{a}+I_{d}\right)}}\right)
$$

With the phase shift between exposures known, the phase of the incident light may be expressed as:

$$
\Delta \phi=\arctan \left(\frac{\sin \Delta \phi}{\cos \Delta \phi}\right)=\arctan \left(\frac{\sqrt{3\left(I_{b}-I_{c}\right)\left(I_{a}-I_{c}\right)}}{\left(I_{b}+I_{c}\right)-\left(I_{a}+I_{d}\right)}\right)
$$

\section{PHASE ERROR AND NOISE}

As with any measurement, there are errors and noise that must be accounted for. In the application of quantitative phase imaging, digital sensors that measure the intensity of incident light are subject to multiple sources of error. These sources of error include photon noise, dark noise, read noise, quantization noise, pixel non-uniformity, as well as electronic interference. Such errors as pixel non-uniformity, and electronic interference are difficult to quantify due to their dependence on factors that are independent to the optical system. As a result, they will only be mentioned here.

Photon noise is a fundamental trait of light and is caused by the discrete nature of photons. This noise is modeled as a Poisson process where the noise is equal to the square root of the intensity of photons incident on the pixel $(\sqrt{I})$.

Dark noise is the false reading of photons by the sensor due to thermally generated electrons on the sensor array. Dark noise is typically specified by the manufacturer of the sensor array and is reported in units of electrons (RMS) per pixel per second of integration.

Read noise is the combinations of all 'on chip' sources of error. In many cameras, on chip processes occur prior to the image data being sent to a computer, the errors introduced as a result of this processing are quantified and combined by sensor and camera manufacturers and reported in units of electrons (RMS) per read.

Quantization noise arises from any analog to digital conversion. In the case of an optical detector, photons are used to generate electrons in each pixel. These electrons build up voltage, which is then counted and converted to a digital number to be stored by the computer. The error of a digitization process has a mean of zero and extrema of $\pm 0.5 L_{S B}$, where $L_{S B}$ is the least significant bit of the analog to digital converter (ADC). The standard deviation of this error is $L_{S B} / \sqrt{12}$ and is commonly used to quantify errors introduced as a result of digitization of an analog signal.

The manufacturing of sensor arrays is a highly controlled and repeatable processes but there are inevitable irregularities in the manufacturing process. Pixel non-uniformity is the error introduced as a result of non-uniform sensitivity to light from one pixel to another on a detector array.

Other errors can be introduced into the measurement of light in the form of electronic interference from nearby uninsulated devices. If a strong enough electronic interference is present prior to the signal amplifiers of the optical sensor, the interference noise will be proportionally amplified and corrupt the signal being measured.

For the following analyses, the error in the measurement of the light intensity incident on a particular pixel will be the root sum square (RSS) off all the contributing noise sources, and will be considered equal between all pixels. Thus, the error of an electric field intensity measurement is:

$$
\delta I_{i}=\sqrt{I_{i}+(\Delta t \delta D)^{2}+M(\delta R)^{2}+\frac{L_{S B}^{2}}{12}}
$$


Where $I_{i}$ is the intensity of photons incident on pixel $i, \Delta t$ is the integration time of the pixel, $\delta D$ is dark noise, $\delta R$ is read noise, and $M$ is the number of reads taken to make the measurement.

\section{Four Bucket/Step Method}

Beginning with the equations for the electric field intensities at four points along a fringe in Equation 4, and its resulting equation for phase in Equation 5, we apply the equation for the propagation of error. The equation for phase variance is thus:

$$
\delta \phi^{2}=\sum_{i=1}^{4}\left(\frac{\partial \phi}{\partial I_{i}}\right)^{2} \delta I_{i}^{2}
$$

The partial differentials of phase with respect to each intensity function are:

$$
\begin{aligned}
& \frac{\partial \phi}{\partial I_{a}}=-\left(\frac{\partial \phi}{\partial I_{c}}\right)=\frac{I_{b}-I_{d}}{\left(I_{a}-I_{c}\right)^{2}+\left(I_{d}-I_{b}\right)^{2}} \\
& \frac{\partial \phi}{\partial I_{b}}=-\left(\frac{\partial \phi}{\partial I_{d}}\right)=\frac{I_{c}-I_{a}}{\left(I_{a}-I_{c}\right)^{2}+\left(I_{d}-I_{b}\right)^{2}}
\end{aligned}
$$

Combining Equations 12, 13a, and 13b, with the expression for intensity errors of Equation 11 yields:

$$
\delta \phi^{2}=\frac{I_{1}+I_{2}+(\Delta t \delta D)^{2}+M(\delta R)^{2}+\frac{L_{S B}^{2}}{12}}{4 I_{1} I_{2}}
$$

This expression may be further reduced by the use of quantities such as fringe visibility and average electric field intensity across a fringe. Fringe Visibility is measure of contrast in the recorded interference pattern by the detector array. Fringe visibility $(V)$ is defined as the amplitude of the fringe divided by the mean fringe value.

$$
V=\frac{2 \sqrt{I_{1} I_{2}}}{I_{1}+I_{2}}
$$

The average electric field intensity across a fringe $(<I>)$ is defined as:

$$
<I>=\frac{\sum_{i=1}^{N} I_{i}}{N}=I_{1}+I_{2}
$$

With Equations 15 and 16, the expression for phase errors introduced by the Four Bucket/Step Method becomes:

$$
\delta \phi=\sqrt{\frac{1}{2 V^{2}<I>}+\frac{(\Delta t \delta D)^{2}+M(\delta R)^{2}+\frac{L_{S B}^{2}}{12}}{2 V^{2}(<I>)^{2}}}
$$

Equation 17, provides an expression for the error propagated by the four bucket/step method as a result of photon, detector, and quantization noise. Note that the above expression consists of two terms on the right-hand side of Equation 17 The left most corresponds to errors as a result of photon noise, which will be present in any optical system due to the discrete nature of light, and the right corresponds to errors that are propagated into the phase measurement as a result of the detector used to make intensity measurements. 


\section{Three Bucket/Step Method}

For the Three Bucket/Step Method, the propagation of error equation is written as:

$$
\delta \phi^{2}=\sum_{i=1}^{3}\left(\frac{\partial \phi}{\partial I_{i}}\right)^{2} \delta I_{i}^{2}
$$

The partial derivatives of phase with respect to the three intensities (Equation 6) are:

$$
\begin{aligned}
& \frac{\partial \phi}{\partial I_{a}}=\frac{I_{b}-I_{c}}{\left(I_{a}-I_{c}\right)^{2}+\left(I_{c}-I_{b}\right)^{2}} \\
& \frac{\partial \phi}{\partial I_{b}}=\frac{I_{c}-I_{a}}{\left(I_{a}-I_{c}\right)^{2}+\left(I_{c}-I_{b}\right)^{2}} \\
& \frac{\partial \phi}{\partial I_{c}}=\frac{I_{a}-I_{b}}{\left(I_{a}-I_{c}\right)^{2}+\left(I_{c}-I_{b}\right)^{2}}
\end{aligned}
$$

Combining Equations 11, 18, 19, as well as the definition of fringe visibility in Equation 15 and average electric field intensity in Equation 16, results in the following expression for phase error due to the three bucket/step method.

$$
\delta \phi=\sqrt{\frac{1+2 \cos ^{2} \phi-2 V \sin (\Delta \phi) \cos ^{2}(\Delta \phi)}{4 V^{2}<I>}+\frac{3\left((\Delta t \delta D)^{2}+M(\delta R)^{2}+\frac{L_{S B}^{2}}{12}\right)(1-\sin (2 \Delta \phi))}{16 V^{2}(<I>)^{2}}}
$$

\section{Carré Method}

Implementing the equation for the propagation of error to the Carré Method, the phase variance is:

$$
\delta \phi^{2}=\sum_{i=1}^{4}\left(\frac{\partial \phi}{\partial I_{i}}\right)^{2} \delta I_{i}^{2}
$$

The partial derivatives of phase with respect to the four recorded intensities defined in Equation 8 are:

$$
\begin{gathered}
\frac{\partial \phi}{\partial I_{a}}=-\left(\frac{\partial \phi}{\partial I_{d}}\right)=\frac{\left(\frac{3\left(I_{b}-I_{c}\right)\left(I_{b}+I_{c}\right)-\left(I_{a}+I_{d}\right)}{2 \sqrt{3\left(I_{b}-I_{c}\right)\left(I_{a}-I_{d}\right)}}\right)+\sqrt{3\left(I_{b}-I_{c}\right)\left(I_{a}-I_{d}\right)}}{\left(\left(I_{b}+I_{c}\right)-\left(I_{a}+I_{d}\right)\right)^{2}+3\left(I_{b}-I_{c}\right)\left(I_{a}-I_{d}\right)} \\
\frac{\partial \phi}{\partial I_{b}}=-\left(\frac{\partial \phi}{\partial I_{c}}\right)=\frac{\left(\frac{3\left(I_{a}-I_{d}\right)\left(I_{b}+I_{c}\right)-\left(I_{a}+I_{d}\right)}{2 \sqrt{3\left(I_{b}-I_{c}\right)\left(I_{a}-I_{d}\right)}}\right)+\sqrt{3\left(I_{b}-I_{c}\right)\left(I_{a}-I_{d}\right)}}{\left(\left(I_{b}+I_{c}\right)-\left(I_{a}+I_{d}\right)\right)^{2}+3\left(I_{b}-I_{c}\right)\left(I_{a}-I_{d}\right)}
\end{gathered}
$$

Because the partial derivatives of $\phi$ with respect to $I_{a}$ and $I_{d}$, as well as $I_{b}$ and $I_{c}$ are odd functions of each other, respectively, Equation 21 can be expressed as:

$$
\delta \phi^{2}=\left(\frac{\partial \phi}{\partial I_{a}}\right)^{2}\left(\delta I_{a}^{2}+\delta I_{d}^{2}\right)+\left(\frac{\partial \phi}{\partial I_{b}}\right)^{2}\left(\delta I_{b}^{2}+\delta I_{c}^{2}\right)
$$

Combining Equations 11, 22, and 23, yields the following expression for phase variance of the Carré Method for phase calculation using an unknown phase shift. 


$$
\begin{aligned}
& \delta \phi^{2}=\frac{A\left(\frac{3 D(B-A)}{2 \sqrt{3 C D}}+\sqrt{3 C D}\right)+B\left(\frac{3 C(B-A)}{2 \sqrt{3 C D}}+\sqrt{3 C D}\right)}{(B-A)^{2}+3 C D} \\
& +\frac{2\left((\Delta t \delta D)^{2}+M(\delta R)^{2}+\frac{L_{S B}^{2}}{12}\right)\left[(3 D(B-A)+6 C D)^{2}+\left((3 D(B-A)+6 C D)^{2}\right]\right.}{12 C D((B-A)+6 C D)^{2}}
\end{aligned}
$$

Where,

$$
\begin{gathered}
A=I_{a}+I_{d}=2<I>+4 \sqrt{I_{1} I_{2}} \cos (\Delta \phi) \cos \left(\frac{3 \alpha}{2}\right) \\
B=I_{b}+I_{c}=2<I>+4 \sqrt{I_{1} I_{2}} \cos (\Delta \phi) \cos \left(\frac{\alpha}{2}\right) \\
C=I_{a}-I_{d}=4 \sqrt{I_{1} I_{2}} \sin (\Delta \phi) \sin \left(\frac{3 \alpha}{2}\right) \\
D=I_{b}-I_{c}=4 \sqrt{I_{1} I_{2}} \sin (\Delta \phi) \sin \left(\frac{\alpha}{2}\right)
\end{gathered}
$$

\begin{tabular}{|c|c|c|c|c|c|}
\hline Sensor & Sensor Type & $\begin{array}{c}\text { Dark Noise } \\
{\left[\mathrm{e}^{-} / \mathbf{s}\right]}\end{array}$ & $\begin{array}{c}\text { Read Noise } \\
{\left[\mathrm{e}^{-/ / \mathrm{read}]}\right.}\end{array}$ & $\begin{array}{c}\text { Well Depth } \\
{\left[\mathrm{e}^{-/} / \text {pixel] }\right.}\end{array}$ & Bit Depth \\
\hline Sony ICX625 & CCD & 8.73 & 0.003 & 5903 & 8 \\
\hline
\end{tabular}

\section{COMPARISONS}

In order to compare the noise characteristics of the four bucket/step, three bucket/step, and Carré Methods, performance metrics from a readily available optical detector were used (Sony ICX625 CCD). The performance metrics for this detected are tabulated in Table 1.

The propagation of error of the four bucket/step method in Equation 17 shows that this phase calculation algorithm introduces random errors. The phase variance as a result of photon, dark, read, and quantization noise is independent of the phase being measured and are thus random for any phase measurement. From this equation, we can also see that the expression for the propagation of the error is inversely proportional to fringe visibility as well as average incident light intensity.

The propagation of error of the three bucket/step method in Equation 20 shows that this phase calculation algorithm introduces systematic errors due to the fact that the phase variance is a function of the phase being measured. Similar to the four bucket/step method, the expression for error in the three bucket/step method is inversely proportional to fringe visibility and average light intensity.

The Carré method introduces a complex expression for the propagation of error due to the fact that the algorithm is based on an interferogram with unknown phase shift. By first having to infer on the phase shift based on direct fringe measurements, errors are compounded. Not only the errors of the intensity measurements of the fringe have to be considered in the calculation of the unknown phase shift $(\alpha)$, but the propagation of errors in the calculation of $\alpha$ propagate to the expression for phase in Equation 10.

All three of the aforementioned phase calculation methods are inversely proportional to both fringe visibility and average electric field intensity recorded by the pixel(s). For digital detectors, such as the Sony ICX625 CCD, with finite pixel well depths, there is a finite number of photons that a pixel can detect before becoming saturated. This imposes an upper limit on the value of the average electric field intensity recorded by the detector $(<I\rangle)$ and thus imposes an upper limit on fringe visibility as pixels begin to saturate. As the average pixel(s) intensity becomes more that $50 \%$ saturated, fringe visibility begins to decrease. As the pixel(s) become fully saturated, the fringe visibility approaches zero making any calculation of phase impossible. 
Figure 1 illustrates the performance of each phase calculation method as (a) a function of normalized average electric field intensity recorded by the detector, and (b) a function of the phase that is to be measured. As mentioned earlier, the three bucket/step method can be employed using various known phase shifts to produce a fringe. The figure depicts the phase errors associated with the three bucket/step method with a phase shift of $\pi / 2$ and $2 \pi / 3$, while the Carré method is employed with a phase shift of $\alpha=\pi$.

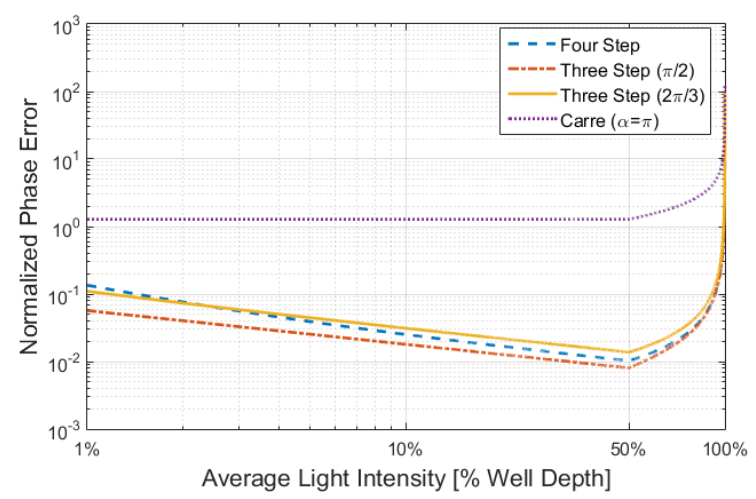

(a)

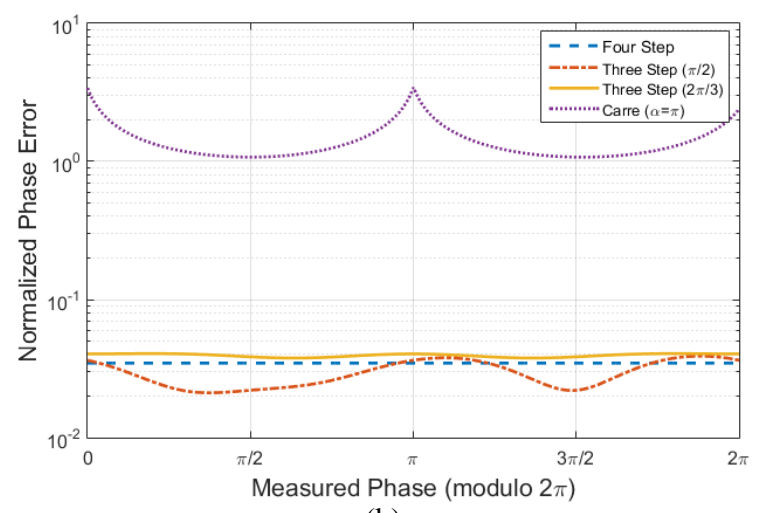

(b)

Figure 1: Comparison of the four bucket/step, three bucket/step, and Carré methods with respect to (a) normalized average light intensity (\% well depth of pixel), and (b) measured phase

\section{EXPERIMENTAL COMPARISONS}

In the implantation of quantitative phase measurements, coherent illumination sources must be used in order to establish interference patterns of the two incident light beams. This introduces speckle noise into the recorded interference pattern. Speckle noise is the localized mutual interference of two coherent illumination sources that interfere either constructively or destructively to produce highly localized bright or dark spots, respectively[16, 17].

To investigate the effects of speckle noise on recorded interferograms, multiple interferograms were recorded using a Sony ICX625 CCD using multiple illumination sources with varying coherence lengths and illumination wavelengths. Speckle noise can be reduced by constricting the coherence length of the illumination source, which reduces the occurrence of the speckles in the interferogram. In quantifying the effects of speckle noise, recorded interferograms were used to calculate local fringe visibilities across the entire field of view of the CCD array. The three illumination sources used were a 405-nm Fabry-Perot benchtop laser source (Thorlabs S1FC405), a 405-nm pigtailed laser coupled diode (Thorlabs LP405-SF10), and a 488-nm fiber coupled diode laser (Toptica iBeam Smart). This 488-nm laser by Toptica allows for tunable coherence lengths in order to attenuate speckle noise.

Figure 2 shows interferograms attained using the aforementioned illumination sources, their respective fringe visibilities across the field of view of the detector as well as a linear intensity plot of fringe visibility showing the coherent envelope of the interferogram.

Speckle noise causes high spatial frequency irregularities in the interference pattern established across an interferogram, which can be seen as high spatial frequency fluctuations in fringe visibility. Of the three illumination sources tested, these fringe fluctuations were quantified by analyzing the variation of fringe visibility through the coherent envelope of the interferogram. Low coherence length illumination sources decrease the occurrence of these speckle patterns thus reducing the spatial variations of fringe visibility across and interferogram.

For the three phase calculation algorithms discussed in this work, as well as many others, phase errors are inversely proportional to fringe visibility. Because of this, variations in fringe visibility caused by speckle noise can directly propagate to the phase reconstruction algorithm thus distorting the quality of the final result. 


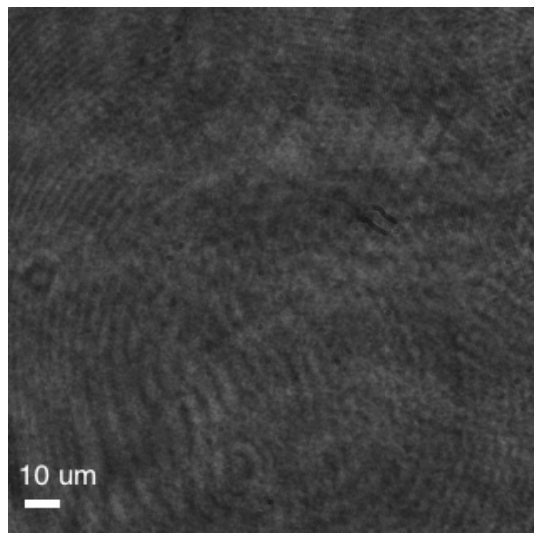

(a)

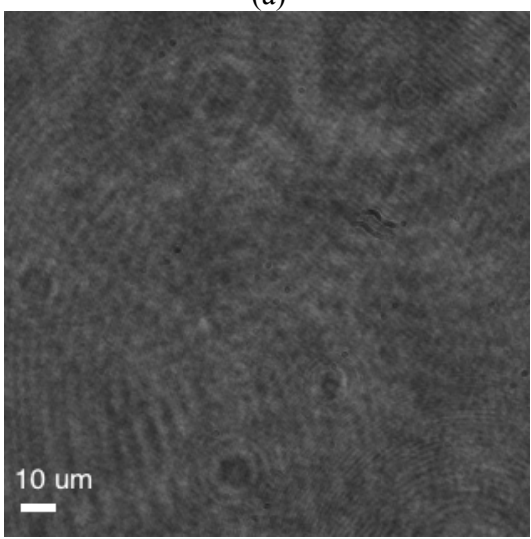

(d)

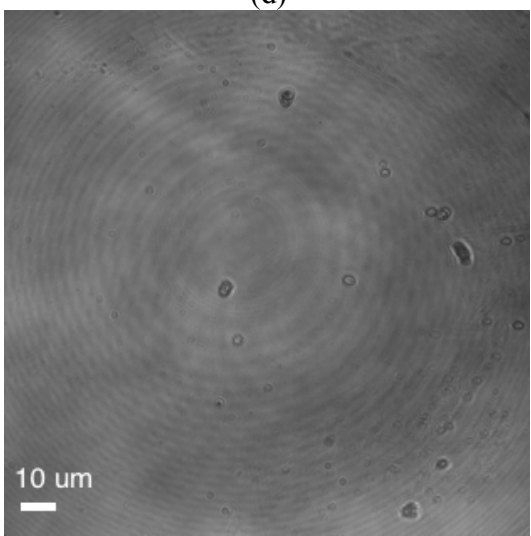

(g)

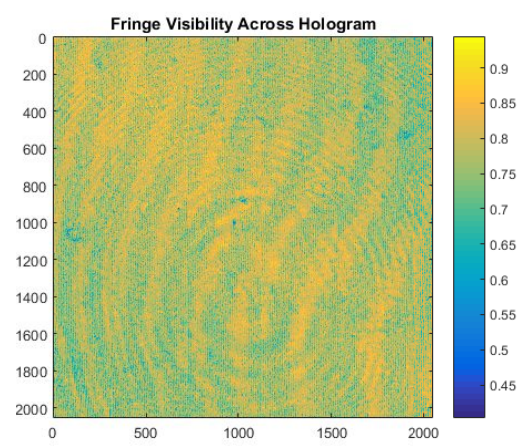

(b)

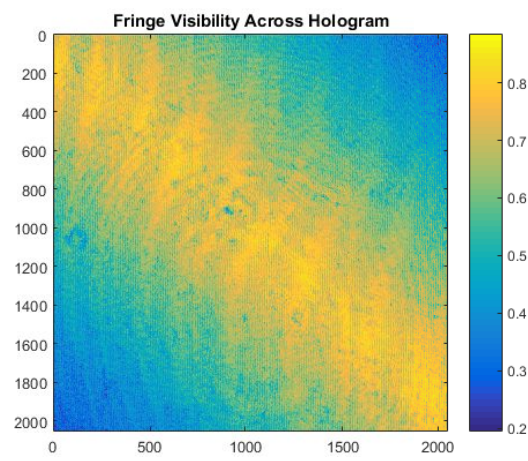

(e)

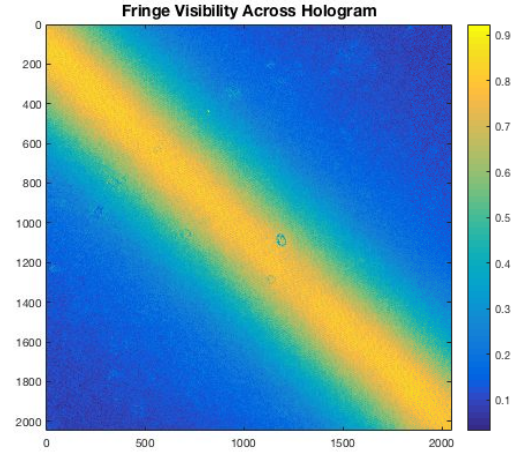

(h)

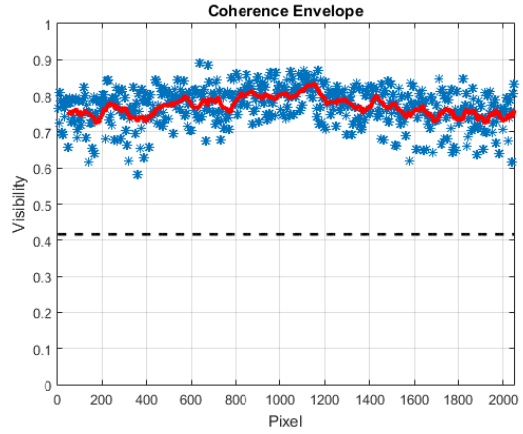

(c)

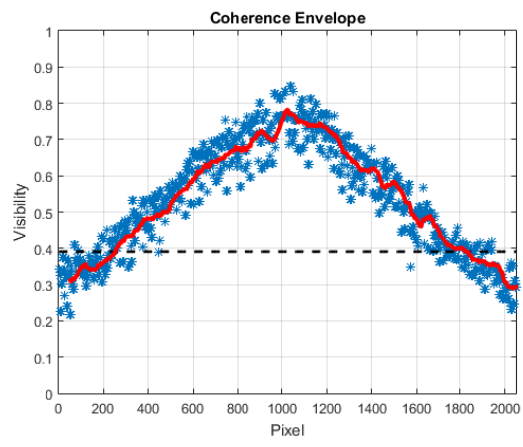

(f)

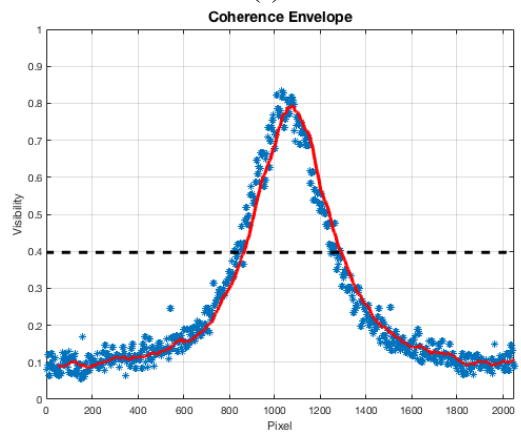

(i)

Figure 2: Analysis of speckle noise using three illumination sources. (a) Interferogram using a long coherence length 405-nm benchtop laser, (b) local fringe visibilities of the interferogram over the entire field of view of the interferogram, and (c) a linear intensity plot of the fringe visibilities showing the coherent envelope of the interferogram. (d) The interferogram obtained by using a 405-nm pig-tailed fiber coupled diode laser, (e) the local fringe visibilities of the interferogram across the entire field of view, and

(f) a linear intensity plot of the fringe visibilities showing the coherent envelope of the interferogram. (g) The interferogram obtained using a low coherence length 488-nm fiber coupled diode laser, (h) the local fringe visibilities of the interferogram across the entire field of view, and (i) a linear intensity plot of the fringe visibilities showing the coherent envelope of the interferogram.

The 405-nm benchtop laser is a long coherence length illumination source and was seen to introduce a relatively large amount of speckle noise, which was seen as high variability of fringe visibility. Fringe visibility varied by $16.3 \%(95 \%$ $\mathrm{CI}$ ), while the shorter coherence length $405-\mathrm{nm}$ pig tailed laser couple diode had a variability in fringe visibility of $10.5 \%(95 \% \mathrm{CI})$, and the 488-nm fiber coupled laser diode had a fringe visibility variability of $7.1 \%(95 \% \mathrm{CI})$. 
The low coherence length 488-nm laser was seen to introduce less speckle noise into the recorded interferogram by a factor of two relative to the benchtop 405-nm diode laser. The decrease in speckle noise comes at a cost, however. By sacrificing coherence length to reduce speckle noise, the usable field of view of the interferogram is reduced by almost $70 \%$. Due to the low coherence length, only a portion of the recorded interferogram is incident to coherent light and so fringe visibility is close to zero for much of the detector array. Along with this, the mode of operation of the low coherence length laser is by the current modulation through the laser diode in order to introduce high temporal frequency incoherence. By doing so, the optical power output of the laser is severely limited making it difficult to use in optical systems that require beam-splitters, which attenuate the intensity of light that is incident on the detector.

\section{CONCLUSION}

Quantitative phase imaging is becoming a widely used technique to measure the optical phase characteristics of objects of interest. Quantitative phase imaging is used in a wide range of disciplines from astronomy to biomedical microscopy. Optical interferometry is a common method that is used to obtained quantitative phase images. Many algorithms exist for the extraction of phase information from interference patterns. Some of the most common are the four bucket/step method, the three bucket/step method, and the Carré method. There has been much development of both pre and post processing algorithms to reduce the noise introduced in such phase measurements, but in this work, we presented a theoretical approach to understand the various sources of noise introduced in a phase measurement as well as how they propagate to the final phase calculation.

Theoretical expressions for the errors introduced by the four bucket/step, three bucket/step and Carré methods were derived and compared to one another. The four bucket/step method was the only method among the three that showed random as opposed to systematic error.

When comparing these three phase calculation techniques, it becomes evident that the four bucket/step or three bucket/step methods are preferable over the Carré method due to their relatively low phase errors. However, the advantage of the Carré method is that a phase measurement can be made without knowledge of the spatial or temporal sampling frequency of the interference pattern.

The propagation of errors between the four bucket/step method and three bucket/step method are comparable to each other, however, the four bucket/step method was shown to introduce random errors which is desirable in applications where a wide range of phases are to be measured. This ensures more consistent performance across all measurements.

Speckle noise was experimentally quantified using three illumination sources with varying coherence lengths. Reductions in coherence length introduces less speckle noise by decrease the occurrence of the random and localized interference of coherent light, but can dramatically decrease the usable field of view of the interferogram. A 488-nm fiber coupled diode laser which uses current modulation to decrease coherent length was seen to introduce a factor of two times less speckle noise than a 405-nm benchtop coherent laser. This decrease in speckle noise severely decreased the usable field of view of the interferogram by having only $30 \%$ of the field of view populated with fringe visibilities sufficient for the calculation of quantitative phase.

\section{REFERENCES}

[1] W. J. Tango and R. Twiss, "IV Michelson Stellar Interferometry," Progress in Optics 17(239-277 (1980)

[2] C. R. Burch and J. P. P. Stock, "Phase-Contrast Microscopy," Journal of Scientific Instruments 19(5), 71 (1942)

[3] $\quad$ F. Zernike, "Diffraction theory of the knife-edge test and its improved form, the phase-contrast method," Monthly Notices of the Royal Astronomical Society 94(377-384 (1934)

[4] T. E. Gureyev and K. A. Nugent, "Rapid quantitative phase imaging using the transport of intensity equation," Optics Communications 133(1-6), 339-346 (1997)

[5] N. Lue, W. Choi, G. Popescu, T. Ikeda, R. R. Dasari, K. Badizadegan and M. S. Feld, "Quantitative phase imaging of live cells using fast Fourier phase microscopy," Applied Optics 46(10), 1836-1842 (2007) 
[6] E. Cuche, P. Marquet and C. Depeursinge, "Spatial filtering for zero-order and twin-image elimination in digital off-axis holography," Appl. Opt. 39(23), 4070-4075 (2000)

[7] K. Creath, "V phase-measurement interferometry techniques," Progress in optics 26(349-393 (1988)

[8] H. Wang, M. Yu, Y. Jiang, Q. Zhu and F. Liu, "Point spread function and lateral resolution analysis of digital holographic microscopy system," Optics Communications 322(90-96 (2014)

[9] Z. Wang, W. Qu, Y. Wen, F. Yang and A. Asundi, "A new phase error compensation method in digital holographic microscopy," in International Conference on Experimental Mechanics 2014, pp. 93023M-93023M-93028, International Society for Optics and Photonics (2015).

[10] F. Pan, W. Xiao, S. Liu, F. Wang, L. Rong and R. Li, "Coherent noise reduction in digital holographic phase contrast microscopy by slightly shifting object," Opt. Express 19(5), 3862-3869 (2011)

[11] Y. Zhang, D. Wang, Y. Wang, Z. Zhou and J. Zhao, "Coherent noise reduction in digital holographic phase contrast microscopy by varying the reference wave," in Smart Nano-Micro Materials and Devices, pp. 82042U-82042U82046, International Society for Optics and Photonics (2011).

[12] T. B. Le, M. Piao, J.-R. Jeong, S.-H. Jeon and N. Kim, "Improving Phase Contrast of Digital Holographic Microscope using Spatial Light Modulator," Journal of the Optical Society of Korea 19(1), 22-28 (2015)

[13] X.-J. Lai, H.-Y. Tu, C.-H. Wu, Y.-C. Lin and C.-J. Cheng, "Resolution enhancement of spectrum normalization in synthetic aperture digital holographic microscopy," Applied optics 54(1), A51-A58 (2015)

[14] M. Matrecano, P. Memmolo, L. Miccio, A. Persano, F. Quaranta, P. Siciliano and P. Ferraro, "Improving holographic reconstruction by automatic Butterworth filtering for microelectromechanical systems characterization," Appl. Opt. 54(11), 3428-3432 (2015)

[15] A. Sharma, G. Sheoran and Z. Jaffery, "Improvement of signal-to-noise ratio in digital holography using wavelet transform," Optics and lasers in engineering 46(1), 42-47 (2008)

[16] P. Memmolo, V. Bianco, M. Paturzo, B. Javidi, P. A. Netti and P. Ferraro, "Encoding multiple holograms for speckle-noise reduction in optical display," Opt. Express 22(21), 25768-25775 (2014)

[17] M. Molaei, M. Barry, R. Stocker and J. Sheng, "Failed Escape: Solid Surfaces Prevent Tumbling of Itextit\{Escherichia coli\}," Physical Review Letters 113(6), 068103 (2014)

[18] F. Charrière, T. Colomb, F. Montfort, E. Cuche, P. Marquet and C. Depeursinge, "Shot-noise influence on the reconstructed phase image signal-to-noise ratio in digital holographic microscopy," Appl. Opt. 45(29), 7667-7673 (2006) [19] N. Demoli, H. Skenderovic and M. Stipcevic, "Digital holography at light levels below noise using a photoncounting approach," Optics Letters 39(17), 5010-5013 (2014) 